\title{
Stimulus-related priming during task switching
}

\author{
MYEONG-HO SOHN and JOHN R. ANDERSON \\ Carnegie Mellon University, Pittsburgh, Pennsylvania
}

\begin{abstract}
Task switch cost (the deficit of performing a new task vs. a repeated task) has been partly attributed to priming of the repeated task, as well as to inappropriate preparation for the switched task. In the present study, we examined the nature of the priming effect by repeating stimulus-related processes, such as stimulus encoding or stimulus identification. We adopted a partial-overlap task-switching paradigm, in which only stimulus-related processes should be repeated or switched. The switch cost in this partial-overlap condition was smaller than the cost in the full-overlap condition, in which the task overlap involved more than stimulus processing, indicating that priming of a stimulus is a component of a switch cost. The switch cost in the partial-overlap condition, however, disappeared eventually with a long interval between two tasks, whereas the cost in the full-overlap condition remained significant. Moreover, the switch cost, in general, did not interact with foreknowledge, suggesting that preparation on the basis of foreknowledge may be related to processes beyond stimulus encoding. These results suggest that stimulus-related priming is automatic and short-lived and, therefore, is not a part of the persisting portion of switch cost.
\end{abstract}

Human action is controlled by both executive mechanisms and automatic mechanisms. The executive mechanisms are goal driven and reflect internal factors, such as intention and prior knowledge. In contrast, the automatic mechanisms are stimulus driven and reflect the strength of stimulus-to-response (SR) associations irrespective of the appropriateness of activating a specific SR association in a given context. Dominance of one type of mechanism over another can create problems in cognitive control. The dominance of the executive mechanisms will result in inflexible processing that does not take detailed features of the stimulus or the event into account. When the automatic mechanisms dominate, the course of action will be constantly changing its direction, making it difficult to achieve goals. Therefore, it is essential to find a balance between these two driving forces.

The purpose of the present study was to examine the effect of stimulus-driven task set priming when people adopt a new task set and how it is affected by goal-driven processes, such as task expectancy. We employed the task-switching paradigm, in which people rapidly alternate between two tasks (task switch) or repeat the same task (task repetition). This paradigm simulates a situation in which a stimulus array can afford multiple task sets. Figure 1 presents an example of a stimulus array that affords a letter task (to

This research was supported by Grant BCS-9975220 from the National Science Foundation. Parts of this research were presented at the 42nd Annual Meeting of the Psychonomic Society, Orlando, FL. We thank Luis Fuentes, Craig Haimson, Nachshon Meiran, and Akira Miyake for their helpful comments on earlier drafts. Correspondence concerning this article should be addressed to M.-H. Sohn, BH345E, Department of Psychology, Carnegie Mellon University, Pittsburgh, PA 15213 (e-mail: mhsohn+@andrew.cmu.edu). judge whether the letter is a consonant or a vowel) or a number task (to judge whether the number is even or odd). The relevant task is indicated by an external cue, such as the stimulus color, or an internal cue, such as a preinstructed sequence. People normally are faster when they repeat the same task than when they switch tasks, a phenomenon called the task switch cost or the task repetition benefit (Jersild, 1927; Spector \& Biederman, 1976).

Several studies have shown that the task switch cost is affected by both top-down executive factors, such as foreknowledge, and bottom-up factors, such as task repetition. The switch cost decreases when people know the identity of an upcoming task in advance and keeps decreasing with a sufficiently long response-to-stimulus interval (RSI) between two tasks (Meiran, 1996; Rogers \& Monsell, 1995). The reduction of the switch cost with foreknowledge reflects increasing executive control processes, such as voluntary preparation for a task switch (De Jong, 2000). However, the switch cost is quite resistant to preparation and decreases only to an asymptotic level without disappearing completely. Also, the switch cost can decrease with factors other than task expectancy. For example, by simply increasing the RSI between two tasks, the switch cost was reduced even when task foreknowledge was not available (Sohn \& Anderson, 2001). This reduction of the switch cost without task expectancy can be interpreted as evidence for automatic activation decay of the recently performed task (Allport, Styles, \& Hsieh, 1994; Allport \& Wylie, 2000; Wylie \& Allport, 2000).

In the present study, we examined the nature of the automatic activation part of switch cost and how it interacts with task expectancy. We focused on stimulus-related priming, which is part of the overall switch cost. In a taskswitching situation, task repetition may take advantage of several kinds of stimulus-related priming. One advantage 


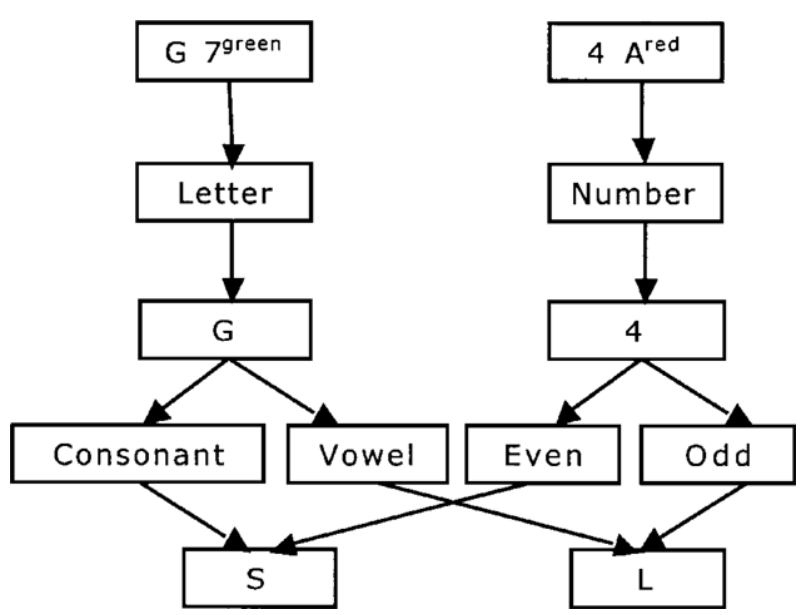

Figure 1. Color-to-task translation and category of stimulusto-response mapping. The superscript indicates the color in which the stimulus was presented.

of task repetition is the task encoding. When the stimulus array affords multiple task sets (see Figure 1), an external or an internal cue has to be provided to indicate the appropriate task set. When there is a task repetition, the task cue is repeated, facilitating the task-encoding process. When there is a task switch, however, a different task cue has to be encoded, and it may take longer to encode that new cue. Another source of advantage for task repetition is the process of identifying the target. Typically in the task-switching paradigm, a distractor accompanies a target. Therefore, it is critical to focus on the target to perform the task properly. In the context of a task repetition, stimuli from the same category are repeatedly identified as targets. However, in a task switch, a stimulus from a different category has to be identified as the target, and it may take longer to do so.

Three research questions are important for stimulusrelated priming. First, is there really a priming effect of the stimulus-related processes, such as stimulus encoding or target identification, when the task repetition does not involve exactly the same stimulus? It has been common practice in the task-switching studies, including the present study, not to repeat the exact stimulus for successive tasks. This is to avoid any possible perceptual facilitation associated with a specific stimulus, so that participants have to process a new stimulus regardless of the task transition. However, perceptual or cognitive priming is not limited to identical stimuli. Priming effects have often been found between categorically associated stimuli, as well as between semantically or associatively related stimuli (Schvaneveldt \& Meyer, 1973). Therefore, it is reasonable to expect that some of the repetition effect may come from repeating stimulus-related processes without exactly repeating the stimulus. However, in a typical taskswitching experiment, it is hard to attribute any effects exclusively to the stimulus-related processes because a fullscale task transition involves repetition or switch of the response-related processes, as well as of the stimulus- related processes. In the present study, we adopted a modified task-switching paradigm, in which tasks were only partially switched or repeated. All the trials consisted of two tasks: Task 1 and Task 2. Task 1, or the prime task, required only task encoding and target identification, whereas Task 2, or the probe task, involved the relevant task (letter or number task) in its entirety. If task encoding or target identification is a source of switch cost, we should obtain a task switch cost even with such a partial overlap between the two tasks.

Second, if there is stimulus-related priming, how long will it last? Perhaps the most interesting result from the task-switching literature is that the switch cost is extremely hard to eliminate. Even with approximately $4,000 \mathrm{msec}$ of RSI, performance still benefits from task repetition (Sohn, Ursu, Anderson, Stenger, \& Carter, 2000). However, as was pointed out earlier, the repetition effect may consist of multiple components. Our partial-overlap task-switching paradigm allowed us to examine the time course of stimulusrelated priming. We used both a short RSI $(500 \mathrm{msec})$, which should result in a sizable switch cost, and a long RSI (2,000 msec), which should result in the residual switch cost only. If stimulus-related priming contributes to the residual switch cost, we should still observe switch cost even with the long RSI. Existing literature, however, indicates otherwise. For example, a switch cost was observed when there were no competing distractors (Meiran, 2000; Rogers \& Monsell, 1995; Ruthruff, Remington, \& Johnston, 2001), suggesting that the stimulus-related processes may not be a critical part of the residual switch cost. If so, we should not observe the residual switch cost in the partial-overlap condition with a long RSI.

Third, is stimulus-related priming independent of intentional preparation? Several studies (Dreisbach, Haider, \& Kluwe, 2002; Ruthruff et al., 2001; Sohn \& Carlson, 2000) have manipulated foreknowledge so that people could prepare for an upcoming task during the RSI in some conditions, but not in others. Results showed a task switch cost with or without foreknowledge, and the amount of the cost remained roughly unaffected by the foreknowledge. With no task expectancy, neither task repetition nor task switch can be systematically prepared, and there should be no difference between task transitions in terms of preparation. Therefore, any difference between task switch and task repetition in this condition should be attributed to the automatic activation benefit that results from repeating the task. The fact that the switch cost was comparable regardless of a chance to prepare for an upcoming task raises a question regarding the nature of the preparation induced by foreknowledge. If foreknowledge does not eliminate stimulus-related priming and yet produces a performance benefit, this implies that preparation may involve processes beyond stimulus encoding or identification. We tested this possibility by examining the switch cost with or without task foreknowledge.

In the experiment, the participants performed two tasks on each trial. Figure 2 illustrates the structure of a trial. On each trial, they performed either a letter task or a number task, with a stimulus array consisting of one letter and 


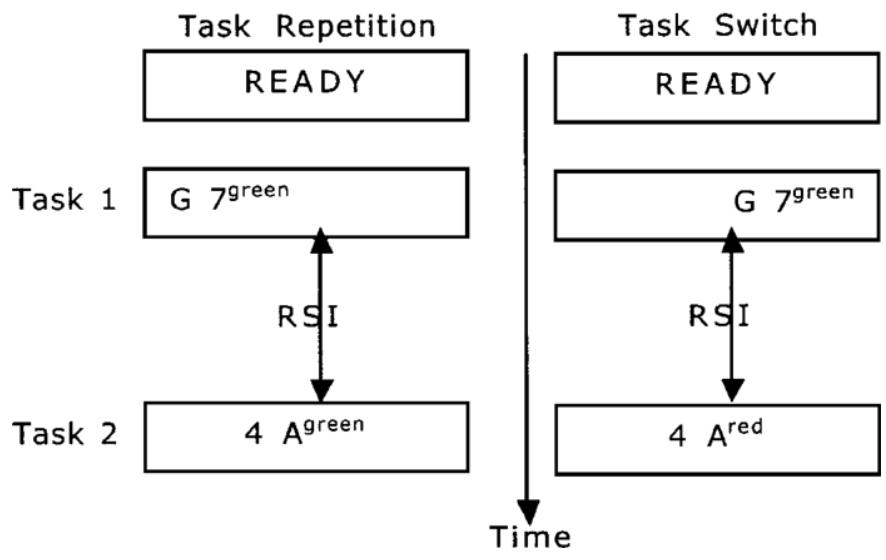

Figure 2. A schem atic of trials.

one number. To examine whether stimulus encoding and target identification could prime performance of the next task, we manipulated the extent of task overlap between Task 1 and Task 2. In the full-overlap condition, Task 1 and Task 2 involved either the letter task or the number task, as described earlier. Therefore, the task repetition or the switch in this condition took place in the entirety of the task. In the partial-overlap condition, Task 1 involved only task encoding and target identification. The Task 1 stimulus consisted of one letter and one number, just as the Task 2 stimulus did, and Task 1 required the same stimulusrelated processes. For example, when the color was green (red), the participants named the presented letter (number). Thus, the participants had to make the connection from green (red) to the letter (number) task and identify the letter (number). However, they did not have to categorize the target further. Also, a verbal response was collected for Task 1. Therefore, in the partial-overlap condition, task switch or repetition was defined only with respect to the stimulus-related processes. We expected that there should be a task switch cost in both the partial-overlap and the full-overlap conditions. However, the switch cost should be smaller in the partial-overlap condition, because the source of benefit is limited to the stimulus.

To examine how long stimulus-related priming persists, we used 500- and 2,000-msec RSIs. The choice of these RSIs was made on the basis of a pilot study, in which the 2,000-msec RSI seemed to be long enough for stimulus priming to dissipate, whereas the 500-msec RSI seemed to be short enough to ensure priming but also long enough to allow preparation on the basis of foreknowledge. In both task overlap conditions, the switch cost should decrease as the RSI increases. By examining the size of the switch cost in different combinations of task overlap and RSI, we would be able to see how the switch cost would decay depending on its sources.

To examine the effect of executive preparation on switch cost, we provided foreknowledge of Task 2 on half of the trials. In the foreknowledge condition, Task 1 was randomly the letter task or the number task, but Task 2 was predictable on the basis of Task 1. For example, the Task 1 stimulus appearing on the left side of the screen indicated that the same task would be repeated in Task 2 . Similarly, the Task 1 stimulus appearing on the right side of the screen indicated a task switch. In the no-foreknowledge condition, task transition was not predictable from where the Task 1 stimulus appeared on the screen.

\section{METHOD}

\section{Participants}

There were four groups of participants ( 83 in total) assigned to the four conditions defined by crossing RSI (500 or 2,000 msec) and task overlap (partial overlap or full overlap). There were 18 in the 500-msec-full-overlap condition, 19 in the 2,000-msec-full-overlap condition, and 23 for each RSI of the partial-overlap conditions. The participants were college students, graduate students, and staff members of Carnegie Mellon University. They participated in return for monetary reward, consisting of a base pay and a bonus proportionate to the speed on correct trials. They received from $\$ 10$ to $\$ 14$ for about a 70-min experimental session.

\section{Task and Equipment}

The stimuli were generated using E-Prime software (Psychology Software Tools, Pittsburgh), and the timing was controlled by a Windows-operated PC. A stimulus consisted of one letter and one number. The letter was randomly selected from four consonants $(\mathrm{G}, \mathrm{K}$, $\mathrm{M}$, and $\mathrm{R}$ ) and four vowels (A, E, I, and U), and the number was randomly selected from four even numbers $(2,4,6$, and 8$)$ and four odd numbers $(3,5,7$, and 9). The left-right order of the letter and the number was randomized, and Task 1 and Task 2 of the same trial did not have any repeated elements. The stimulus array was presented in green or red. The green color indicated the letter task, and the red color indicated the number task. The "s" and the "l" keys were used to make responses. The even and the consonant responses were assigned to one key, and the odd and the vowel responses were assigned to the other, with the response-to-key mapping counterbalanced across participants. When the task was target identification in the partialoverlap conditions, a voice key was used to take a vocal response.

\section{Procedure and Design}

As is shown in Figure 2, a trial began with the READY signal at the center of a computer screen. This signal lasted for $500 \mathrm{msec}$, and a blank screen replaced it for $500 \mathrm{msec}$. Then the Task 1 stimulus followed and remained on the screen until the participant made a response or until 1,000 msec had elapsed, whichever occurred first. The Task 1 stimulus appeared either on the left or the right side of 
the screen. The position of the Task 1 stimulus served as a cue for foreknowledge when it was available. In the full-overlap conditions, Task 1 involved the letter task or the number task in their entirety. In the partial-overlap conditions, Task 1 was to identify the target and say it out loud. The delay between the Task 1 response and the Task 2 stimulus presentation (RSI) was either 500 or $2,000 \mathrm{msec}$. The Task 2 stimulus also remained on the screen until the participant pressed a response key or until 1,000 msec had elapsed, whichever occurred first. The Task 2 stimulus was presented at the center of the screen. At the end of each trial, feedback was given on accuracy and latency for both Task 1 and Task 2 . The trial-by-trial feedback was given so that the participants could follow the instruction to give an equal emphasis to accuracy and speed and also an equal emphasis to Task 1 and Task 2 .

An experimental session began with a practice block of 32 trials, in which the participants were exposed to all possible task transitions in a random order. In the main experiment, there were 16 blocks of 16 trials. Task foreknowledge was provided in 8 blocks (foreknowledge condition), but not in the other 8 blocks (no-foreknowledge condition). The participants were informed whether foreknowledge would be available in a particular block. The foreknowledge and the no-foreknowledge blocks alternated. The resulting design was a 2 (partial and full task overlap) $\times 2(500-$ and 2,000 -msec RSIs $) \times 2$ (foreknowledge and no-foreknowledge) $\times 2$ (task repetition and task switch) mixed factorial. Task overlap and RSI were manipulated between groups.

\section{RESULTS}

A trial was counted as correct when both Task 1 and Task 2 responses were correct. Only correct trials were included in the Task 2 latency analysis. Two participants from the 2,000-msec RSI-full-overlap condition did not meet the accuracy criterion (.70), so their data were discarded from the analyses below. The switch cost in our paradigm was defined as the difference in Task 2 latency between switch and repetition trials. Therefore, we analyzed accuracy and Task 2 latency, applying a four-way analysis of variance (ANOVA) with task overlap (full or partial), RSI (500 and 2,000 $\mathrm{msec}$ ), foreknowledge (foreknowledge and no-foreknowledge), and task transition (repeat and switch) as factors.

\section{Accuracy}

Mean accuracies are presented in Table 1. Accuracy was higher with task repetition (.91) than with task switch $\left[.88 ; F(1,77)=52.10, M S_{\mathrm{e}}=0.001, p<.0001\right]$, higher with foreknowledge (.91) than with no foreknowledge $\left[.89 ; F(1,77)=10.08, M S_{\mathrm{e}}=0.002, p<.01\right]$, and higher with partial task overlap (.91) than with full task overlap $\left[.88 ; F(1,77)=4.78, M S_{\mathrm{e}}=0.014, p<.05\right]$. The effect of task transition (repeat or switch) interacted with task overlap $\left[F(1,77)=4.96, M S_{\mathrm{e}}=0.001, p<.05\right]$ and RSI $\left[F(1,77)=9.58, M S_{\mathrm{e}}=0.001, p<.01\right]$. The switch cost was greater with full task overlap (.04) than with partial task overlap $[.02 ; t(79)=2.12, p<.05]$ and was greater with a 500-msec RSI (.04) than with a 2,000 msec RSI $[.02 ; t(79)=3.23, p<.01]$. No other main effects or interactions were significant $(p>.10)$. The pattern of accuracy mirrors the pattern of latency, described next.

\section{Task 1 Latency}

Our primary measure was Task 2 latency, because that should reflect the effects of the variables of interest. The analysis of Task 1 latency can provide some insights into how participants prepare for Task 2 . For example, with foreknowledge, Task 1 provides information regarding Task 2. Therefore, Task 1 performance may be affected by the information conveyed by foreknowledge. Because Task 1 for the partial-overlap conditions was different from Task 1 for the full-overlap conditions, we analyzed Task 1 latency from each condition separately and conducted three-way ANOVAs with transition (repetition or switch), foreknowledge (foreknowledge and no foreknowledge), and RSI (500 and 2,000 msec).

In the partial-overlap conditions, Task 1 latency was longer with foreknowledge $(919 \mathrm{msec})$ than with no foreknowledge [899 msec; $F(1,44)=7.41, M S_{\mathrm{e}}=2,490, p<$ $.01]$, presumably reflecting the preparation on the basis of foreknowledge. This Task 1 slowdown due to foreknowledge was evident only in the 500-msec RSI condition $(39 \mathrm{msec})$ and not in the 2,000-msec RSI condition [ $2 \mathrm{msec}$; $\left.F(1,44)=6.31, M S_{\mathrm{e}}=13,312, p<.05\right]$. Also, this slowdown was evident only when Task 1 was embedded within a task switch (35 msec), and not within a task repetition [6 msec; $\left.F(1,44)=6.43, M S_{\mathrm{e}}=1,411, p<.05\right]$. In the full-overlap conditions, the Task 1 performance was slower when Task 1 conveyed foreknowledge $(1,574 \mathrm{msec})$ than when it did not $\left[1,524 \mathrm{msec} ; F(1,33)=5.27, M S_{\mathrm{e}}=\right.$ $21,866, p<.05]$. The Task 1 slowdown due to foreknowledge was evident only in the 2,000-msec RSI condition $(114 \mathrm{msec})$, and not in the 500-msec RSI condition [ $1 \mathrm{msec}$; $\left.F(1,44)=5.16, M S_{\mathrm{e}}=21,866, p<.05\right]$. Similar to the partial-overlap condition, the Task 1 slowdown due to foreknowledge was greater within a task switch $(80 \mathrm{msec})$ than within a task repetition $(29 \mathrm{msec})$, but this effect was only marginally significant $(p<.07)$. In summary, Task 1

Table 1

Mean Accuracies as a Function of Condition

\begin{tabular}{|c|c|c|c|c|c|c|c|c|}
\hline \multirow[b]{3}{*}{ Condition } & \multicolumn{4}{|c|}{ Partial Task Overlap } & \multicolumn{4}{|c|}{ Full Task Overlap } \\
\hline & \multicolumn{2}{|c|}{ 500-msec RSI } & \multicolumn{2}{|c|}{ 2,000-msec RSI } & \multicolumn{2}{|c|}{ 500-msec RSI } & \multicolumn{2}{|c|}{ 2,000-msec RSI } \\
\hline & $M$ & $S E$ & $M$ & $S E$ & $M$ & $S E$ & $M$ & $S E$ \\
\hline Repetition with foreknowledge & .94 & .01 & .93 & .02 & .92 & .02 & .90 & .02 \\
\hline Switch with foreknowledge & .89 & .01 & .93 & .01 & .88 & .02 & .87 & .02 \\
\hline Repetition with no foreknowledge & .92 & .01 & .91 & .02 & .91 & .02 & .89 & .02 \\
\hline Switch with no foreknowledge & .89 & .01 & .91 & .01 & .85 & .02 & .86 & .02 \\
\hline
\end{tabular}

Note-RSI, response-to-stimulus interval. 

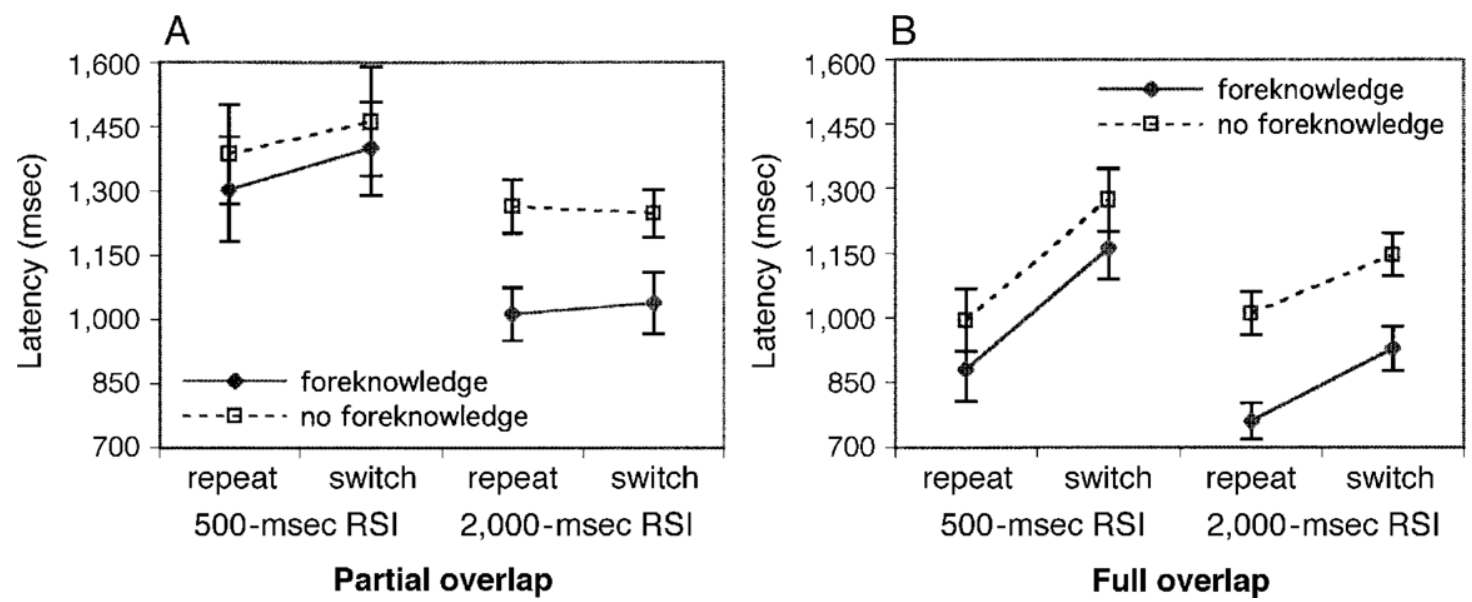

Figure 3. Mean Task 2 latencies as a function of task transition, foreknowledge, and response-to-stimulus interval (RSI). Panel A is partial overlap, and panel B is full overlap.

latency provided evidence that people seem to prepare for Task 2 when foreknowledge is available even during the performance of Task 1 .

\section{Task 2 Latency}

Figure 3 shows the mean Task 2 latency as a function of task transition, foreknowledge, and RSI. The Task 2 latency was shorter with task repetition $(1,076 \mathrm{msec})$ than with task switch $\left[1,208 \mathrm{msec} ; F(1,77)=102.94, M S_{\mathrm{e}}=13,312\right.$, $p<.0001]$, shorter with foreknowledge $(1,061 \mathrm{msec})$ than with no foreknowledge $[1,223 \mathrm{msec} ; F(1,77)=82.04$, $\left.M S_{\mathrm{e}}=25,656, p<.0001\right]$, shorter with full overlap $(1,020 \mathrm{msec})$ than with partial overlap [1,265 msec; $\left.F(1,77)=8.77, M S_{\mathrm{e}}=54,1670, p<.01\right]$, and shorter with the 2,000-msec RSI $(1,146 \mathrm{msec})$ than with the 500-msec RSI $\left[1,343 \mathrm{msec} ; F(1,77)=15.00, M S_{\mathrm{e}}=541,670, p<\right.$ $.0001]$.

Several two-way interactions were significant. The foreknowledge effect was greater with the 2,000-msec RSI $(232 \mathrm{msec})$ than with the $500-\mathrm{msec}$ RSI $(93 \mathrm{msec})$, resulting in a significant foreknowledge $\times$ RSI interaction $\left[F(1,77)=4.82, M S_{\mathrm{e}}=25,656, p<.05\right]$. This interaction indicates that task preparation increased as more time was permitted. The main effect of transition (switch cost) also significantly interacted with $\operatorname{RSI}[F(1,77)=16.05$, $\left.M S_{\mathrm{e}}=13,312, p<.0001\right]$ and with task overlap $[F(1,77)=$ 44.01, $\left.M S_{\mathrm{e}}=13,312, p<.0001\right]$. The last two interactions are critical in this study, so they were further investigated in terms of the switch cost (the difference between task switch and task repetition), plotted in Figure 4. A two-way ANOVA was conducted with task overlap and RSI as variables. The switch cost was greater with full task overlap $(217 \mathrm{msec})$ than with partial overlap [45 msec; $\left.F(77)=44.01, M S_{\mathrm{e}}=13,312, p<.0001\right]$. This result indicates that the switch cost consists of multiple components and that stimulus-related priming is one of them. The switch cost was also greater with the 500-msec RSI $(183 \mathrm{msec})$ than with the 2,000-msec RSI [79 msec; $\left.F(77)=16.05, M S_{\mathrm{e}}=13,312, p<.01\right]$. Moreover, the switch cost with the 2,000-msec RSI in the partial-overlap condition $(5 \mathrm{msec})$ was not significantly different from 0 $(p>.60)$, whereas the costs from other conditions were significantly different from 0 ( $p$ s $<.05)$. This result indicates that part of the switch cost decays over time and that the component based solely on stimulus-related priming may completely decay away.

\section{DISCUSSION}

The aim of the present study was to understand how executive and automatic control mechanisms interact in a task-switching situation. For executive influence, we examined the task foreknowledge effect; for automatic influence, we examined the priming effect of stimulus-related processes and compared it with processes that go beyond stimulus encoding and target identification. In some conditions of the present study, to isolate the stimulus-related processing, we modified the traditional task-switching paradigm so that only part of a task was switched or repeated. We also examined the effect of foreknowledge. Although foreknowledge resulted in a significant task per-

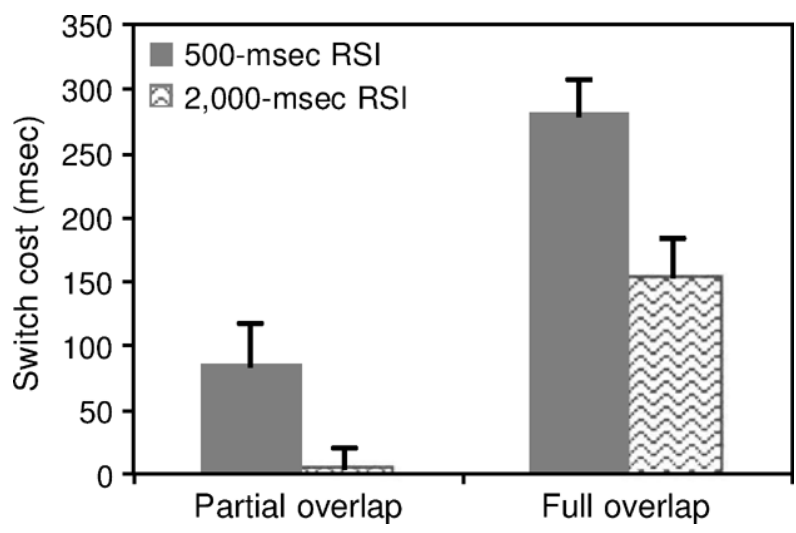

Figure 4. Switch costs in each condition of response-to-stimulus interval (RSI) and task overlap. 
formance benefit, it did not interact with any variable other than RSI. The interaction with RSI was quite expected, because preparation on the basis of foreknowledge needs time to build up (Neely, 1977).

Switch cost, however, interacted with variables other than foreknowledge. Switch cost was greater with a short RSI than with a long RSI and was greater when task overlap involved the entire task than when it involved part of the task. We will conclude with several implications of these results for the nature of switch cost. First, switch cost does seem to consist of multiple components, with the stimulus-related processes being one important set of components. The idea that switch cost reflects multiple processes is consistent with the current view of switch cost. For example, Meiran, Chorev, and Sapir (2000) showed that the benefit of task repetition consists of decreasing components, such as passive dissipation of the previous task set and increasing preparation for a new task set, as well as persisting components known as residual switch cost.

Second, stimulus-related priming seems to be shortlived. The switch cost with a 2,000-msec RSI disappeared in the partial-overlap condition, but not in the full-overlap condition, suggesting that the source of the residual effect observed in the full-overlap condition may not be attributed to stimulus-related processes. Meiran (2000) showed that switch cost disappeared at long RSIs, when competing tasks did not share response sets. However, as long as there was ambiguity to be resolved in terms of response key mappings, switch cost persisted even when the stimulus array afforded only one task set, so that there was no competition between different tasks at the stimulus level. The present study also provides evidence, although indirectly, that response-related processes, such as response selection and execution, may be responsible for the persisting switch cost.

Third, as can be seen in Figure 3, the foreknowledge effect does not interact with task overlap, implying that stimulus-related priming is indeed automatic and not under direct control of executive mechanisms. This result has implications for the nature of the preparation effect on the basis of foreknowledge in a task-switching situation. When available, foreknowledge does not indicate the identity of the upcoming stimulus but only of the upcoming task at an abstract level. Therefore, it is not possible that preparation could involve anticipation of a specific stimulus. Preparation may evoke a process that is not directly related to the stimulus but, rather, to higher level control, such as shifting goals (Rubinstein, Meyer, \& Evans, 2001) or strengthening task demands of a particular task (Gilbert \& Shallice, 2002). For example, foreknowledge reduces the number of possible SR mapping rules. In the present study, foreknowledge can indicate that only two, not four, SR mapping rules would be relevant for the upcoming task. On this basis, participants could prepare a reduced number of SR mapping rules.

In sum, this study demonstrated that limiting the scope of task overlap can successfully isolate a component of a switch cost-namely, stimulus priming. The stimulus priming is automatic, and not under the control of executive mechanisms, and eventually disappears with a sufficient time intervening between two tasks. Moreover, foreknowledge did not modulate stimulus-related priming, suggesting that foreknowledge may allow preparation at a rather higher level.

\section{REFERENCES}

Al 1 port , D. A., St yl es, E. A., \& Hsieh, S. (1994). Shifting intentional set: Exploring the dynamic control of tasks. In C. Umiltà \& M. Moscovitch (Eds.), Attention and performance XV: Conscious and nonconscious information processing (pp. 421-452). Cambridge, MA: MIT Press, Bradford Books.

Al 1 port, D. A., \& Wyl ie, G. R. (2000). Task-switching, stimulus-response bindings, and negative priming. In S. Monsell \& J. Driver (Eds.), Control of cognitive processes: Attention and performance XVIII (pp. 3570). Cambridge, MA: MIT Press.

De Jong, R. (2000). An intention-activation account of residual switch costs. In S. Monsell \& J. Driver (Eds.), Control of cognitive processes: Attention and performance XVIII (pp. 357-376). Cambridge, MA: MIT Press.

Dreisbach, G., Haider, H., \& Kl uwe, R. H. (2002). Preparatory processes in the task switching paradigm: Evidence from the use of probability cues. Journal of Experimental Psychology: Learning, Memory, \& Cognition, 28, 468-483.

Gil bert, S. J., \& Shall ice, T. (2002). Task switching: A PDP model. Cognitive Psychology, 44, 297-337.

Jersil d, A. T. (1927). Mental set and shift. Archives of Psychology, Whole No. 89.

Meiran, N. (1996). Reconfiguration of processing mode prior to task performance. Journal of Experimental Psychology: Learning, Memory, \& Cognition, 22, 1423-1442.

Meir an, N. (2000). Reconfiguration of stimulus task-sets and response task-sets during task switching. In S. Monsell \& J. Driver (Eds.), Control of cognitive processes: Attention and performance XVIII (pp. 377 399). Cambridge, MA: MIT Press.

Meir an, N., Chor ev, Z., \& Sa pir, A. (2000). Component processes in task-switching. Cognitive Psychology, 41, 211-253.

Neel y, J. H. (1977). Semantic priming and retrieval from lexical memory: Role of inhibitionless spreading activation and limited capacity attention. Journal of Experimental Psychology: General, 106, 226-254.

Roger s, R. D., \& Monsel 1, S. (1995). Costs of a predictable switch between simple cognitive tasks. Journal of Experimental Psychology: General, 124, 207-231.

Ru binst ein, J. S., Meyer, D. E., \& Evans, J. E. (2001). Executive control of cognitive processes in task switching. Journal of Experimental Psychology: Learning, Memory, \& Cognition, 27, 763-797.

Rut hr uff, E., Remingt on, R., \& Johnst on, J. C. (2001). Switching between simple cognitive tasks: The interaction of top-down and bottomup factors. Journal of Experimental Psychology: Human Perception \& Performance, 27, 1404-1419.

Sch van evel dt, R., \& Meyer, D. E. (1973). Retrieval and comparison processes in semantic memory. In S. Kornblum (Ed.), Attention and performance IV (pp. 421-452). New York: Academic Press.

Sohn, M.-H., \& An der son, J. R. (2001). Task preparation and task repetition: Two-component model of task switching. Journal of Experimental Psychology: General, 130, 764-778.

Sohn, M.-H., \& Carl son, R. A. (2000). Effects of repetition and foreknowledge in task-set reconfiguration. Journal of Experimental Psychology: Learning, Memory, \& Cognition, 26, 1445-1460.

Sohn, M.-H., Ur su, S., Ander son, J. R., St en ger, V. A., \& Cart er, C. S. (2000). The role of prefrontal cortex and posterior parietal cortex in task-switching. Proceedings of the National Academy of Sciences, 97, 13448-13453.

Spect or, A., \& Bieder man, I. (1976). Mental set and shift revisited. American Journal of Psychology, 89, 669-697.

Wyl ie, G. R., \& Al 1 port, D. A. (2000). Task switching and the measurement of "switch costs." Psychological Research, 63, 212-233.

(Manuscript received July 15, 2002;

revision accepted for publication March 10, 2003.) 